\title{
Wake-Up Radio systems for Cooperative-Intelligent Transport Systems architecture
}

\author{
Jordi Casademont \\ Universitat Politècnica de Catalunya \\ i2CAT Foundation \\ Barcelona, Spain
}

\author{
Elena Lopez-Aguilera \\ Universitat Politècnica de Catalunya \\ Castelldefels, Spain
}

\author{
Josep Paradells \\ Universitat Politècnica de Catalunya \\ i2CAT Foundation \\ Barcelona, Spain
}

\begin{abstract}
Cooperative-Intelligent Transport systems are new applications developed on top of communications between vehicles and between vehicles and fixed infrastructure. Their architecture envisages devices deployed along the routes and streets, transmitting and receiving different kind of messages belonging to different services. Quite often, these devices will be located in isolated places with very low number of vehicles passing nearby. Being in isolated places, these devices will require to be feed with rechargeable batteries and alternative power sources, the usage of which need to be very efficient. The fact of continuously transmitting messages whenever there is no vehicle to receive them demands a solution. In this paper, we propose to use a well-known saving power strategy already used in Internet of Things, the Wake-up Radio systems. As vehicular communications are based on IEEE 802.11 standard, we propose to use a Wake-up Radio system based on this standard as well, being thus no additional hardware needed for the wake-up transmitter. The paper analyses the feasibility of using this solution on several vehicular applications.
\end{abstract}

Keywords-C-ITS, WuR, Wake-up, LTE-V2X, 802.11p, 5G, IoT, connected vehicles.

\section{INTRODUCTION}

Internet of Things (IoT) expands in many diverse fields and communication between vehicles, understood in the broadest of the senses (cars, trucks, trams but also bikes and even walking persons), being one of the most promising for technical development and market perspectives.

Since the early 2000 s, different standardization bodies have been issuing standards for the so called Cooperative Intelligent Transport Systems (C-ITS). Those from ETSI (European Telecommunications Standards Institute) in Europe, SAE (Society of Automotive Engineers), IEEE (Institute of Electrical and Electronics Engineers) and NTCIP (National Transportation Communications for ITS Protocol) in USA or ISO (International Organisation for Standardisation) worldwide, are the most adopted, but standards issued in Japan or Korea are also relevant in their respective regions. About 200 standards are being prepared to define all aspects of services and protocols for communications Vehicle-to-Vehicle (V2V), Vehicle-toInfrastructure (V2I) and Infrastructure-to-Vehicle (I2V) [1].

Some of these new services use only V2V communication, however, many of them have to rely on fixed infrastructure, the so-called Road Side Units (RSU). This is the case of services as roadworks warning, in-vehicle signage, travel information, infrastructure warning, etc.

When it comes to analysing the scenarios where these RSUs will work, we can foresee urban environments or highways with high volume of traffic, but also RSUs will be deployed in rural roads or small villages, with very few passing vehicles, and even less if we consider night time. Nevertheless, C-ITS services have been designed to continuously broadcast their information messages at frequencies between $1 \mathrm{~Hz}$ and $10 \mathrm{~Hz}$ [2]. This continuous transmission tax may not represent any problem for RSUs connected to electrical power sources, but it may be a quite critical problem for those ones that are isolated in the middle of the countryside or on mountain roads, mounted on poles of traffic signals and powered by batteries with solar panels. It is even made worse if we think that most of the time nobody will receive this information for the lack of vehicles.

On the other hand, in Wireless Sensor Networks (WNS), another primary field of IoT, researchers have large experience on techniques to save energy consumption [3], [4]. There are different approaches, the first to be implemented relies on duty-cycling, that is, periodical activation followed by a sleep period of the radio interface of the wireless sensor node. Unfortunately, if applied to try to save energy on RSUs for vehicular communications on depopulated roads, RSUs would still have to transmit their information on active periods with no vehicle in the vicinity, and having sleep periods not too large, consuming in this way large amount of energy unnecessarily. Moreover, when one vehicle is in the coverage area of the RSU, it will receive discontinuous transmissions according to the duty cycle, and therefore, penalize the performance of the C-ITS service.

The alternative to duty cycle algorithms are Wake-up Radio (WuR) systems. In WuR, an additional, simplistic, hardware receiver, the so-called Wake-up Receiver (WuRx), is attached to an interrupt-capable General-Purpose InputOutput (GPIO) pin of the microcontroller unit (MCU) of the sensor node. This MCU is configured to remain in its lowest power mode by default. The purpose of the WuRx is to detect a Wake-up Call (WuC) sent by a Wake-up Transmitter (WuTx) in the remote nodes prior to any wireless data communication. Upon such $\mathrm{WuC}$ detection, the WuRx generates an interrupt to the node's MCU to which it is attached and causes it to switch from sleep to active mode. Next, the MCU activates the main wireless radio interface, and the participating nodes may proceed to communicate in a traditional fashion. If we apply this technology to RSUs, we would be able to awake them only when vehicles are nearby, and the flow of $\mathrm{I} 2 \mathrm{~V}$ messages will be continuous while the vehicle is in the coverage area of the RSU, thus solving the problems explained before and drastically reducing energy consumptions.

In this paper, we analyse the possibility of using WuR systems on RSUs powered by batteries belonging to a C-ITS architecture within areas of low or very low traffic. The paper is organized as follows. Section II presents the components, protocols and radio technologies of vehicular networks. Section III introduces the use cases where this proposal could be used and their requirements. Section IV analyses WuR solutions, their performance and evaluates if they comply with the previous requirements. Finally, Section $\mathrm{V}$ concludes the paper and presents some final recommendations for using WuR systems on RSUs. 


\section{C-ITS SYSTEMS}

C-ITS are vital to increase safety and tackle traffic's growing emission and congestion problems. They can make transport safer, more efficient and sustainable by applying various information and communication technologies to all modes of passenger and freight transport. Moreover, C-ITS systems will provide platforms in which third party service providers can exploit their own initiatives and deploy a wide range of different applications and services. These services and applications require a functional architecture of system elements and a communication protocol architecture.

\section{A. C-ITS Architecture}

C-ITS have four main connected elements (Fig. 1):

- Vehicular ITS station: Device installed in vehicles and also called On Board Unit (OBU).

- Personal ITS station: Smartphones and other devices whose objective is to assist the so-called Vulnerable Road Users or VRU (cyclists, pedestrians, ...). Usually, these stations are also called personal OBU.

- Roadside ITS station: Devices installed next to the road and called Road Side Unit (RSU).

- Central ITS station: Traffic management centres and back-office of service providers.

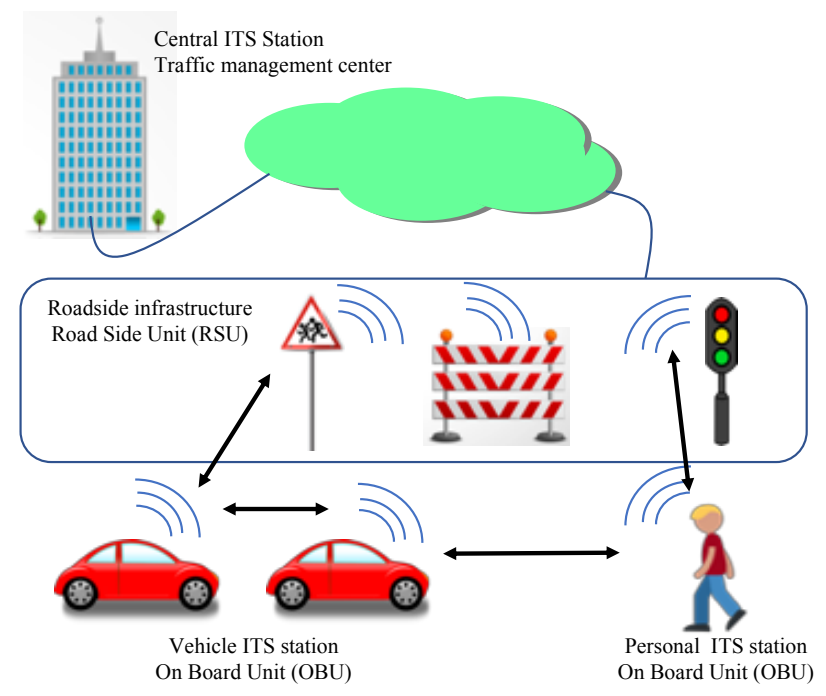

Fig. 1. C-ITS infrastructure elements.

In this distributed V2I architecture, vehicle's OBUs communicate with the infrastructure present in positions close to the road (RSUs). There are different types of RSUs:

- Connected RSUs: These RSUs are connected to management centres or back-offices through a communications network and are intended to be deployed in places where they can be plugged to the electric power grid. Their basic functions are to receive messages from nearby vehicles and send them to the central ITS and forward messages from the management centres to vehicles. Nevertheless, response time for an eventual request/response or information/actuation action between vehicle and centre is quite large. Thus, it is possible to upgrade this architecture connecting RSUs to close Mobile Edge Computing (MEC), which can perform further message forwarding or security functions.
- Isolated RSUs: In many situations, when deploying RSUs in countryside or mountain roads, it will be impossible, or very expensive, to connect them to the electric power grid and therefore, these RSUs will have to work with rechargeable batteries and alternative power sources. It is also possible that these RSUs are connected to central ITS through cellular networks. The situation of these RSUs is that they will be used only from time to time and with long periods without any vehicle passing nearby. It is in this situation that we propose the usage of $\mathrm{WuR}$ technologies, similar to those already in use in different scenarios of IoT.

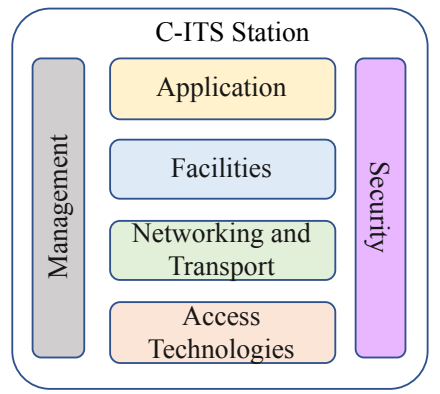

Fig. 2. C-ITS communication protocols architecture.

\section{B. C-ITS Communication Protocol Architecture}

There are several communication protocols architectures, which, more or less, agree on the same structure that is designed on six elements (Fig. 2):

- Application layer provides end user applications.

- Facilities layer provides support functions for applications and the definition of standard messages that can be used by different applications.

- Networking and transport layer provides data routing functions (single-hop or multi-hop), identification of source and destination applications, data congestion management and error control.

- Access technologies layer provides radio Medium Access Control (MAC) and physical levels. It allows different technologies being IEEE 802.11p and LTE$\mathrm{V} 2 \mathrm{X}$ the ones with more projection nowadays.

- Management provides management functions to the device and applications.

- Security provides functions such as encryption, authentication, authorization and firewall.

\section{Radio technologies for C-ITS}

Frequency band [5855 - 5925] $\mathrm{MHz}$ has been allocated worldwide for C-ITS radio communications. As an example, Table I shows the characteristics of the different channels in Europe [5]. There is one control channel (G5-CCH) that is the default transmitting/receiving channel, and six service channels (G5-SCH1 to G5-SCH6). It is also possible to use the outdoors band of IEEE $802.11 \mathrm{a} / \mathrm{n} / \mathrm{ac}$ between [5470 5725] $\mathrm{MHz}$, which is called channel G5-SCH7. Nevertheless, this band requires Transmission Power Control and Dynamic Frequency Selection, which is not possible to use in vehicular communications. This band can be used, for instance, for V2I communications in a garage. 
TABLE I. European channels for C-ITS communications [5]

\begin{tabular}{|c|c|c|c|c|c|}
\hline $\begin{array}{c}\text { Channel } \\
\text { type }\end{array}$ & $\begin{array}{c}\text { IEEE } \\
\text { channel } \\
\text { number }\end{array}$ & $\begin{array}{c}\text { Centre } \\
\text { freq. } \\
\text { (MHz) }\end{array}$ & $\begin{array}{c}\text { Channel } \\
\text { spacing } \\
\text { (MHz) }\end{array}$ & $\begin{array}{c}\text { TX power } \\
\text { limit }(\mathrm{dBm} \\
\text { EIRP) }\end{array}$ & $\begin{array}{c}\text { Default } \\
\text { data rate } \\
\text { (Mbits/s) }\end{array}$ \\
\hline G5-CCH & 180 & 5900 & 10 & 33 & 6 \\
\hline G5-SCH1 & 176 & 5880 & 10 & 33 & 6 \\
\hline G5-SCH2 & 178 & 5890 & 10 & 23 & 12 \\
\hline G5-SCH3 & 174 & 5870 & 10 & 23 & 6 \\
\hline G5-SCH4 & 172 & 5860 & 10 & 0 & 6 \\
\hline G5-SCH5 & 182 & 5910 & 10 & 0 & 6 \\
\hline G5-SCH6 & 184 & 5920 & 10 & 0 & 6 \\
\hline G5-SCH7 & 94 to & 5470 to & several & 30 & several \\
\hline
\end{tabular}

Up to present day, IEEE 802.11p has been the de facto wireless technology standard for vehicle-to-everything (V2X) communications since its release in 2010. It is the former access technology of the Wireless Access in Vehicular Environment (WAVE) protocol architecture in the US, and it has also been adopted in the ETSI ITS-G5 for Europe. It is a relatively mature technology and has already been validated by over a decade of field trials. Nevertheless, theoretical and simulation studies show high level of errors under heavy traffic conditions [6], [7].

Regarding the technology, IEEE 802.11p uses CarrierSense Multiple Access with Collision Avoidance (CSMA/CA). In fact, it is a slight variation of the widely used IEEE 802.11a (known as OFDM in IEEE Std 802.112016) removing the requirement of having to be associated to a Basic Service Set (BSS) before being able to transmit or receive data. For this reason, IEEE $802.11 \mathrm{p}$ is called "Outside the Context of a BSS" (OCB). Its distributed and uncoordinated access makes it an ideal technology to be used in vehicular networks. Nevertheless, collisions due to hidden terminal situations represent a quite high resource waste.

The physical layer uses the same modulation and coding schemes as IEEE $802.11 \mathrm{a}$, but channel bandwidth is of 10 $\mathrm{MHz}$ instead of $20 \mathrm{MHz}$. Therefore, data rates are the half as well, beginning at $3 \mathrm{Mbps}$ and reaching up to $27 \mathrm{Mbps}$, being $6 \mathrm{Mbps}$ the default data rate. Besides, while IEEE $802.11 \mathrm{a}$ works at frequencies between [5150 - 5730] $\mathrm{MHz}$, IEEE 802.11p uses the band [5855 - 5925] MHz (Table I).

In order to renew this standard, the new study group from IEEE named IEEE 802.11 Next Generation V2X was started in March 2018, and evolves to IEEE 802.11bd Task Group (TGbd) in January 2019. Its objectives are to define at least one mode that achieves at least 2 times higher throughput than as in IEEE Std 802.11-2016 operating at maximum mandatory data rate as defined in the $5.9 \mathrm{GHz}$ band (12 Mbps in a $10 \mathrm{MHz}$ channel), in high mobility channel environments at vehicle speeds up to $250 \mathrm{~km} / \mathrm{h}$; to define at least one mode that achieves at least $3 \mathrm{~dB}$ lower sensitivity level than that of the lowest data rate defined in IEEE Std 802.11-2016 operating in $5.9 \mathrm{GHz}$ band; and to define procedures for at least one form of positioning in conjunction with V2X communications [8].

Long-Term Evolution (LTE) based V2X from the Third Generation Partnership Project (3GPP) is a relatively new alternative to IEEE $802.11 \mathrm{p}$-based V2X communications. The first version of LTE-V2X was published in June 2017 under Release 14, which came with numerous enhancements to the existing Device-to-Device (D2D) communications in order to accommodate vehicular communications. The proposed enhancements include a new arrangement of the resource grid of the physical layer, and two types of D2D channel access mechanisms: i) a mechanism coordinated by the evolved NodeB (eNB), named Mode 3, and ii) a distributed mechanism, where User Equipments (UEs) access the channel on their own, named Mode 4. Moreover, LTE-V2X employs different radio interfaces: i) interface between the vehicle and eNB, named LTE-Uu, and ii) interface between vehicles, named PC5. PC5 is intended for transmission of messages belonging to C-ITS applications. $\mathrm{Uu}$ is used for vehicles to request transmission resources on PC5 channel to eNB, and for eNB to grant these resources to vehicles, so the cellular network selects and manages the radio resources used by vehicles for their direct $\mathrm{V} 2 \mathrm{~V}$ communications, thus enabling maximum collision reduction. Mode 4 includes a distributed scheduling scheme for vehicles to select their radio resources.

While LTE-V2X Mode 4 only requires PC5 channel, which can be allocated on G5-CCH or on G5-SCHn, it is not clear where channel LTE-Uu for the connection between vehicles and eNB of Mode 3 will be allocated. Nonetheless, LTE-V2X higher flexibility contributes to a higher reliability and range with respect to IEEE 802.11p [6].

At present day, it is uncertain which technology will lead the marked and, for now, manufacturers are preparing their OBU/RSU shipped with both, IEEE 802.11p and LTE-V2X.

\section{C-ITS messages}

C-ITS protocol architecture defines the Facilities layer which harmonizes messages used by the Application layer. In this way, the number of used messages is maintained low although the number of applications could be large. ETSI and SAE International have defined several messages; the most important are as follows:

- CAM (Cooperative Awareness Message) is a message exchanged between ITS stations to create and maintain awareness of each other and to support cooperative performance of vehicles using the road network. A CAM contains status and attribute information of the originating ITS station.

- DENM (Decentralised Environmental Notification Message) is mainly used by ITS applications in order to alert road users of a detected event as accident, roadworks, obstacles on the road, slow vehicle, dangerous curve, ...

- SPATEM (Signal Phase and Timing Extended Message) provides real-time information of the traffic lights signal phase and timing of an intersection.

- MAPEM (MAP -topology- Extended Message) provides the topology/geometry of a set of lanes. It is transmitted jointly with SPATEM to indicate the geographic situation of the traffic lights whose light signal phases are announced.

- IVIM (Infrastructure to Vehicle Information Message) announces mandatory and advisory road signage such as contextual speeds and road works warnings. IVIM either provides information of physical road signs such as static or variable road signs, virtual signs or road works.

- SREM (Signal Request Extended Message) is sent by an OBUs to RSUs for requesting traffic light signal 
priority (public transport) or signal pre-emption (public safety).

- SSEM (Signal request Status Extended Message): In response to the request, RSUs acknowledge with a SSEM notifying if the request has been granted, cancelled or changed in priority.

- RTCMEM (Radio Technical Commission for Maritime services Extended Message) enables several types of position corrections (GPS, GLONAS, RTK) using correction data generated by road side equipment (stationary GNSS Base station) and used for correction in receiving mobile stations (vehicles).

Some of these messages are sent periodically and others only under specific circumstances. Focussing on those that are sent periodically, normal vehicles only send CAM, emergency vehicles send CAM plus DENM, and infrastructure RSUs have to periodically send CAM, DENM (when there is some special event), SPATEM, MAPEM, IVIM and RTCMEM.

As we can notice, RSUs have to transmit a large number of messages, even if there is no vehicle nearby. In the next section, we analyse which are the requirements of the different C-ITS applications and the feasibility that RSUs powered by rechargeable batteries with some source of external power harvesting as solar panels or wind generators, use $\mathrm{WuR}$ systems as a way to optimize energy consumption.

\section{C-ITS APPLICATIONS AND THEIR REQUIREMENTS}

There is a wide range of different applications that will evolve according to the communication network performance. References from ETSI [8] and [9] provide a first extensive set of use cases, which we can classify according to the way of communication:

- Infrastructure to vehicle (I2V): in-vehicle signage, roadworks warning, applications utilising traffic light phase time information, travel information, ...

- Vehicle to infrastructure (V2I): floating car data and identification of traffic conditions, signal priority or pre-emption request, ...

- Vehicle to vehicle (V2V): hazard warning, collision warning, emergency electronic brake light alert, intersection warning, co-operative driving, ...
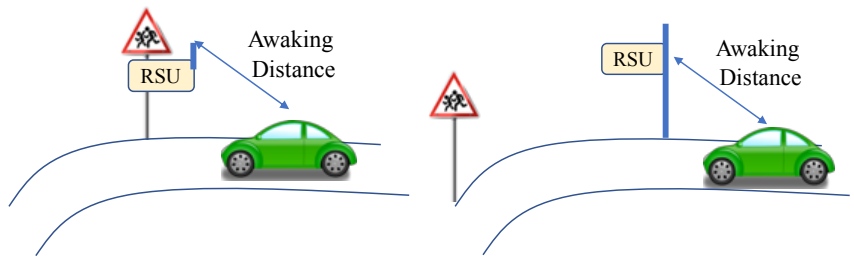

Fig. 3. Distance from traffic signal and RSU to the vehicle.

In our case of study, we are basically interested on applications that transmit messages from the infrastructure on isolated places where the volume of traffic is low or nonexistent for long periods of time. In these cases, isolated RSUs could make use of the WuR systems to improve the lifetime of their batteries. However, as the coverage distance of WuR systems is much shorter than IEEE 802.11p or LTE$\mathrm{V} 2 \mathrm{X}$, the considered services that can make use of this system should not have critical distance requirements (Fig. 3 left). Nevertheless, if do exist a distance requirement, it is always possible to deploy the RSU some distance before the traffic signal as in Fig. 3 right. Section IV analyses awaking distances of wake-up technologies.

ETSI has recently issued a technical specification focused on services provided by the infrastructure [10]. Most of these services require the RSU to be connected to the management centre using a backbone communication network. Thus, in our proposal we assume that RSUs will have communication devices with the backbone network that can be awakened using the trigger message of the wake-up receiver device. Based on these specifications, the different ITS services whose isolated RSUs could make use of the WuR systems are as follows:

- Traffic signage broadcasting service: RSUs near traffic signals transmit IVIMs notifying information about the signal, which can be variable (different speed limits for day and night, icy road on winter, ...) or static (dangerous curve, intersection, STOP, ...). It would be desirable to receive IVIM from one traffic signal from 30 to 100 meters before the signal.

- Transmission service of DENMs ordered by the traffic management centre: This use case is quite similar to the previous one but, while IVIM is associated to a traffic signal with more or less permanent conditions, DENM is associated to an eventual situation, for instance slow vehicle, accident, adverse weather condition, roadworks, ... As the temporal event can be at different distances from the RSU reporting it, this application has no critical distance requirements.

- Traffic Light Maneuver or Green Light Optimal Speed Advisory service: In this application, RSUs transmit SPATEM and MAPEM in order to notify the position and phases of traffic lights. With this information, vehicles calculate the optimal speed to reach the traffic light on green state. If the RSU is deployed in the traffic light it will not require WuR because it can be powered from the electrical grid but, if the RSU is deployed some distance before the traffic light, in a place without electrical power connection, it can make use of the WuR system and there will not have critical distance requirements.

- Traffic Light Control service: It enables vehicles to request priority in intersections controlled by traffic lights using SREM. When vehicles pass besides RSUs they request priority for traffic lights that are some distance away. As in the previous application, it has no critical distance requirements if the RSU is deployed some distance before the traffic light.

- Transmission service by RSUs of CAMs and DEMNS generated by other vehicles and forwarded by the backbone ITS infrastructure: These messages are informative but not critical because transmitting vehicles are far away from receiving vehicles. For instance, one vehicle near the referred RSU receives information that an emergency vehicle is approaching but is still several kilometres away. This application has no critical distance requirements.

- Reception service by RSUs of CAMs and DENMs generated from nearby vehicles and forward them to ITS management centres: Management centres use 
these messages to be aware of especial situations and collect statistics. This application has no critical distance requirements.

- GNSS positioning correction service: It consists in the transmission of RTCMEM providing position correction information for systems like GPS, GLONAS, RTK. This application has no critical distance requirements.

\section{WAKE-Up RADIO For C-ITS ROAD SIDE UNITS}

As introduced in Section I, WuR systems are intended to reduce energy consumption. For this purpose, WuR solutions are built of two radio interfaces. The primary transceiver is in an energy-saving state and is used for main communication, whereas the secondary receiver consists in a permanently active low-power receiver and is employed to activate the main interface upon reception of a $\mathrm{WuC}$ (Fig. 4).

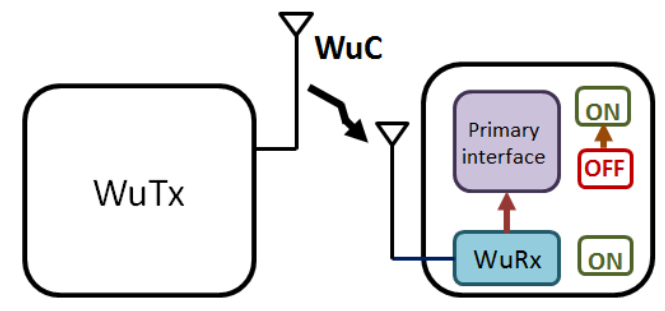

Fig. 4. WuR System.

WuR solutions use mainly radio frequency (RF) signals, however, there have been some acoustic and infra-red based proposals in the literature. There are WuR solutions where the address of a specific destination node or a group of nodes can be indicated, and where the address and communication settings can be configurable. Also there are WuR proposals based on existing RF technologies. In this case, specifically, there are some WuR proposals based on IEEE 802.11 technology in the literature. Legacy IEEE 802.11 Beacon frames have been employed for WuC generation in [12]. In this work, WuRx wakes up the main radio upon energy detection over a threshold. The main advantage of this proposal is the usage of legacy IEEE 802.11 transmitters for $\mathrm{WuC}$ generation. However, system is prone to interferences showing a high number of false positive detections. With the objective of reducing this effect, [13] introduces an address pattern. In this work, the transmission of long legacy IEEE 802.11 data frames alternates with silence periods, thus generating on and off periods, respectively, in order to code address patterns following a Non-Return-to-Zero (NRZ) pattern. This system is still prone to interferences due to the presence of long silence periods. In a different approach [14], length of legacy IEEE 802.11 data frames is used to code the address pattern. The proposal also includes several mechanisms to reduce the number of false positive detections. In this case, however, the WuRx is in the $\mathrm{mW}$ order, with this value being too high for this kind of lowpower receivers. In [15] a WuR system is presented with the WuC composed of a single IEEE 802.11 frame with modified OFDM symbols to build the address pattern. The main disadvantage of this proposal is that it cannot be implemented using legacy IEEE 802.11 transmitters, and it has been validated only employing simulations. In a similar way, in [16] the $\mathrm{WuC}$ is built by controlling the data pattern of OFDM subcarriers and obtaining an amplitude modulated signal. Again, its implementation is unfeasible with legacy
IEEE 802.11 transmitters. In [17] an On-Off-Keying (OOK) WuC built from a single IEEE 802.11 frame is presented with the WuRx consuming $95 \mu \mathrm{W}$. In this case, a firmware update is necessary in legacy IEEE 802.11 transmitters. Finally, in [18] we propose a complete $\mathrm{WuR}$ system with a low-power WuRx consuming $10 \mu \mathrm{W}$. For the WuTx, an offthe-shelf legacy IEEE 802.11 transmitter is employed without any firmware or hardware modification. An OOK $\mathrm{WuC}$ is generated with on periods composed of the transmission of multiple legacy IEEE 802.11 broadcast frames, and with off periods built with silence intervals. The proposal in [18] has been evaluated in indoors and outdoors providing a coverage range of up to $40 \mathrm{~m}$ in both scenarios. In December 2016, new IEEE 802.11ba task group (TGba) started working on forthcoming PHY and MAC layer specifications for WuR operation [19]. TGba proposes the use of one single but modified IEEE 802.11 frame as WuC, thus increasing achievable bit rate. OOK modulation with Manchester coding is employed in the payload field, being this approach not backwards compatible with legacy IEEE 802.11 devices. In order to overcome this issue, in [20] we propose a technique to generate a pseudo-OOK modulation using one single legacy IEEE 802.11 frame by means of choosing the input bitstream to the OFDM PHY. From the implementation proposals presented in previous references, aside from reference [18], only reference [16] provides range detail, being it of $15 \mathrm{~m}$ in indoors.

As IEEE $802.11 \mathrm{p}$ technology is being employed in V2X communications, with IEEE $802.11 \mathrm{p}$ always present in OBUs, it is advisable to propose a WuR system for C-ITS based on IEEE 802.11p technology. Its PHY layer is based on IEEE 802.11a specification, thus changing frequency band, signal bandwidth and bit rate, but preserving characteristics such as OFDM utilization with respect to IEEE 802.11 mainstream specifications. Also, main MAC features are preserved. In this way, based on the exposed state of the art for IEEE 802.11-based WuR systems, we propose to use the IEEE 802.11p transmitter at OBUs as WuTx to send a WuC built of IEEE 802.11p frames, such as in [18]. In any case, proposed WuR solution for C-ITS is not limited to IEEE 802.11-based WuR system presented in [18]. Forthcoming IEEE 802.11-based WuR solutions being backwards compatible with legacy IEEE 802.11 devices and leading to a sufficient coverage distance can be used instead.

The low-power WuRx has to be set at RSUs powered by batteries, which upon detection of the $\mathrm{WuC}$ proceeds to wake up the primary radio interface at RSUs, being this primary interface either IEEE $802.11 \mathrm{p}$ or LTE-V2X. The delay for the wake-up procedure is below $1 \mathrm{~ms}$, being this value low enough for the correct reception of C-ITS messages (Section II.D) sent with a frequency between $1 \mathrm{~Hz}$ and $10 \mathrm{~Hz}$.

Another important feature in a WuR system for C-ITS is $\mathrm{WuC}$ operating range, as maximum available distance between OBUs and RSUs will condition the requirements of $\mathrm{C}$-ITS services. In order to extent the coverage range of WuR system, G5-CCH and G5-SCH1 channels are candidates to be used for $\mathrm{WuC}$ transmission, as the highest EIRP value of up to $33 \mathrm{dBm}$ can be employed within both channels (Table I). However, it is advisable to keep G5-CCH idle, as it is the control channel and, thus, the default transmitting/receiving channel used to announce available services. In this way, the most suitable possibility is to use G5-SCH1 service for WuC transmission. 
Based on the presented state of the art, we consider the range of $40 \mathrm{~m}$ provided in [18] as our reference for maximum operating distance. Proposal in [18] provides evaluation at $2.4 \mathrm{GHz}$ with $20 \mathrm{dBm}$ EIRP. On the other hand, IEEE 802.11 p operates at $5.9 \mathrm{GHz}$ with up to $33 \mathrm{dBm}$ EIRP. The higher frequency band leads to larger propagation losses, however, this effect is in some extent compensated through the increase in the transmitted power. Following this reasoning, we believe $40 \mathrm{~m}$ is a reasonable reference for range value. Nevertheless, in case of requirement of larger distances, it would also be possible to consider active WuRx systems, that although their consumption is higher, their action range is also longer.

In the following we provide a discussion on the feasibility of the WuR system for C-ITS with regards to the services presented in Section III. Traffic signage broadcasting consists in the single service, from the seven presented in Section III, having distance restrictions (30 to $100 \mathrm{~m}$ before the signal) and, thus, being affected by the operating range of $\mathrm{WuR}$ solution. However, in this case, the recommendation is to deploy the RSUs to wake up not in the same traffic signals, but some distance before corresponding traffic signals (recall Fig. 3 right), so that RSUs are awakened in a coverage range of up to $40 \mathrm{~m}$ without affecting traffic signage broadcasting traffic service. For the remaining six services, distance requirements have been seen as non-critical for the concrete applications, as the RSUs are not deployed together with the traffic signals, but some distance in advance (i.e., Traffic Light Maneuver or Green Light Optimal Speed Advisory service, Traffic Light Control service, and Transmission service by RSUs of CAMs and DEMNs generated by other vehicles and forwarded by the backbone ITS infrastructure), or even RSUs are independent from traffic signals at all (i.e., Transmission service of DENMs ordered by the traffic management centre, Reception service by RSUs of CAMs and DENMs generated from nearby vehicles and forward them to ITS management centres, and GNSS positioning correction service).

\section{CONClusions}

In this paper we have analysed the feasibility of using a Wake-up Radio system to solve the problem of energy waste due to the transmission of C-ITS messages from isolated RSUs when there is no vehicle nearby to receive them. We propose to use a WuR system based on IEEE 802.11, which provides a coverage of up to $40 \mathrm{~m}$, with a low-power WuRx consuming $10 \mu \mathrm{W}$ and a delay for the WuR procedure below $1 \mathrm{~ms}$. Using IEEE 802.11-based WuR systems has the advantage that, as manufacturers are preparing their OBUs to be shipped with both IEEE 802.11p and LTE V2X interfaces, it will not be necessary to add extra hardware on vehicles to build the wake-up transmitter. We have reached to the conclusion that most of the services provided from the infrastructure are able to work with RSUs awaken by passing vehicles using WuR systems.

\section{ACKNOWLEDGMENTS}

This work was partially supported by the ERDF and the Spanish Government through project TEC2016-79988-P, AEI/FEDER, UE; and by Secretaria d'Universitats i Recerca del Departament d'Empresa i Coneixement de la Generalitat de Catalunya through project 2017 SGR 00376.

\section{REFERENCES}

[1] D. Green, C. Karl, F. Faber, "Cooperative Intellingent Transport Systems (C-ITS). Estandards assessment". Research report AP-R47415. Austroads. January 2015.

[2] ETSI TR 102638 V1.1.1. "Intelligent Transport Systems (ITS); Vehicular communications; Basic set of applications; Definitions". ETSI. June 2009.

[3] J. Oller, I. Demirkiol, J. Casademont, J. Paradells, G. Ulrich, L. Reindl, "Has time come to switch from duty-cycled MAC protocols to Wake-up Radio for Wireless Sensor Networks?", IEEE/ACM Transactions on Networking, vol. 24, no. 2, pp. 674-687, April 2016.

[4] F. Zahra Djiroun, D. Djenouri, "MAC protocols with Wake-up Radio for Wireless Sensor Networks: A review", IEEE Communications surveys \& tutorials, vol. 19, no. 1, 2017.

[5] ETSI EN 302663 V1.2.1. "Intelligent Transport Systems (ITS); Access layer specification for Intelligent Transport Systems operating in the $5 \mathrm{GHz}$ frequency band", ETSI, July 2013.

[6] A. Bazzi, B. M. Masini, A. Zanella and I. Thibault, "On the Performance of IEEE $802.11 \mathrm{p}$ and LTE-V2V for the Cooperative Awareness of Connected Vehicles", IEEE Transactions on Vehicular Technology, vol. 66, no. 11, pp. 10419-10432, November 2017.

[7] R. Molina-Masegosa and J. Gozalvez, "LTE-V for sidelink 5G V2X vehicular communications", IEEE Vehicular Technology Magazine, vol. 12, no. 4, pp. 30-39, December 2017.

[8] IEEE 802.11, "802.11 NGV proposed PAR", Status of IEEE 802.11 Next Generation V2X SG, November 2018.

[9] ETSI TR 102638 V1.1.1, "Intelligent Transport Systems (ITS); Vehicular Communications; Basic Set of Applications; Definitions", June 2009.

[10] ETSI TS 102 637-1 V1.1.1, " Intelligent Transport Systems (ITS); Vehicular Communications; Basic Set of Applications; Part 1: Functional Requirements", September 2010.

[11] ETSI TS 103301 V1.2.1, "Intelligent Transport Systems (ITS); Vehicular Communications; Basic Set of Applications; Facilities layer protocols and communication requirements for infrastructure services", August 2018.

[12] N. Mishra, K. Chebrolu, B. Raman, A. Pathak, "Wake-on-WLAN," in Proc. of International Conf. on World Wide Web, 2006.

[13] N. Mishra, D. Golcha, B. Raman, K. Chebrolu, "S-WOW: Signature based Wake-on-WLAN," in Proc. of International Conf. on Communication Systems Software and Middleware, 2007.

[14] S. Tang, H. Yomo, Y. Takeuchi, "Optimization of frame length modulation-based Wake-up control for green WLANs," IEEE Transactions on Vehicular Technology, vol. 64, no. 2, pp. 768-780, February 2015.

[15] F. Hutu, A. Khoumeri, G. Villemaud, J. M. Gorce, "Wake-up radio architecture for home wireless networks," in Proc. of IEEE Radio and Wireless Symposium, 2014.

[16] H. Zhang, C. Li, S. Chen, X. Tan, N. Yan, H. Min, "A low power OFDM-based wake-up mechanism for IoT applications," IEEE Transactions on Circuits and Systems II: Express Briefs, vol. 65, no. 2, pp. 181-185, February 2018.

[17] E. Alpman, A. Khairi, R. Dorrance, M. Park, V. Somayazulu, "802.11g/n compliant fully integrated Wake-up Receiver with $72 \mathrm{dBm}$ sensitivity in 14nm FinFET CMOS," IEEE Journal of SolidState Circuits, vol. 53, no. 5, pp. 1411-1422, May 2018.

[18] J. Oller, E. Garcia, E. Lopez-Aguilera, I. Demirkol, J. Casademont, J. Paradells, U. Gamm, L. Reindl, "IEEE 802.11-enabled wake-up radio system: design and performance evaluation", Electronics Letters, vol. 50, no. 20, pp. 1484-1486, September 2014.

[19] TGba, "IEEE Standard for Information Technology, Local and Metropolitan Area Networks, Specific Requirements, Part 11: Wireless LAN Medium Access Control and Physical Layer Specifications Amendment 9: Wake-up Radio Operation" IEEE P802.11 WG, IEEE Standard Draft P802.11ba/D2.0, January 2019.

[20] M. Cervia, A. Calveras, E. Garcia-Villegas, E. Lopez-Aguilera, I. Demirkol, J. Paradells, "Doc IEEE 802.11-19/0138r0. An extension to 11ba: legacy IEEE 802.11 transmitter solution for $802.11 \mathrm{ba}$ receivers", $15^{\text {th }}$ January 2019, IEEE 802.11 WG, https://mentor.ieee.org/802.11/dcn/19/11-19-0138-00-0wng-anextension-to-11ba-legacy-ieee-802-11-transmitter-solution-for-80211ba-receivers.pptx 